\title{
Endoscopic closure of acute Boerhaave's syndrome with an over-the-scope clip
}

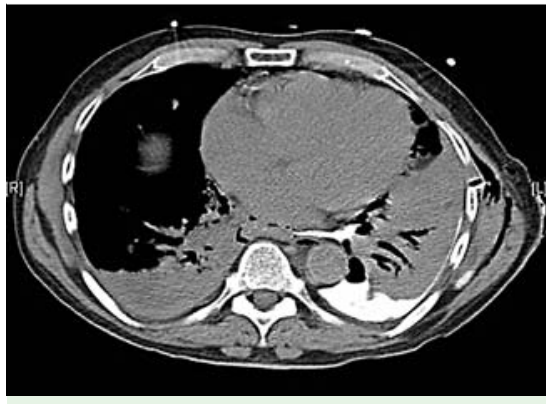

Fig. 1 Computed tomography scan showing contrast leak into the left pleural space from the esophagus.

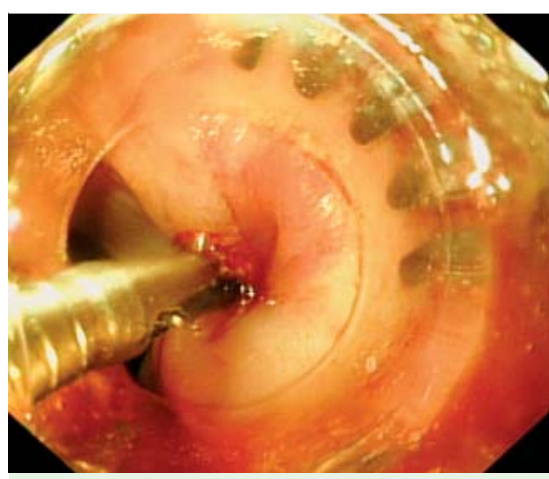

Fig. 4 Twin grasper retracting the edges of the perforation.

A 63-year-old man attended the accident and emergency department with chest pain and breathlessness following an episode of severe vomiting after excess alcohol intake. Clinical examination and chest radiography confirmed a left-sided tension pneumothorax requiring an emergency thoracocentesis and chest drain placement. Computed tomography (CT) scan with intravenous contrast showed a left-sided pneumothorax and pneumomediastinum, which suggested the possibility of an esophageal rupture in the absence of external trauma. A CT scan with oral contrast performed subsequently, showed leakage of contrast from the lower esophagus into the left pleural space, consistent with lower esophageal leak due to Boerhaave's syndrome ( $\bullet$ Fig. $\mathbf{1})$. After fluid resuscitation, administration of antibiotics, and stabilization in intensive

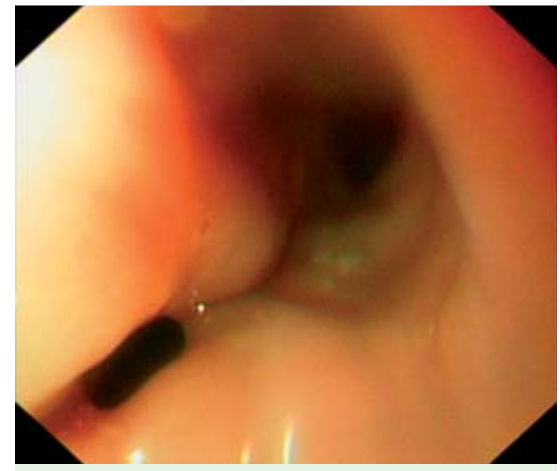

Fig.2 Endoscopic view of esophageal perforation with fistula at the 7 o'clock position (Day 3).
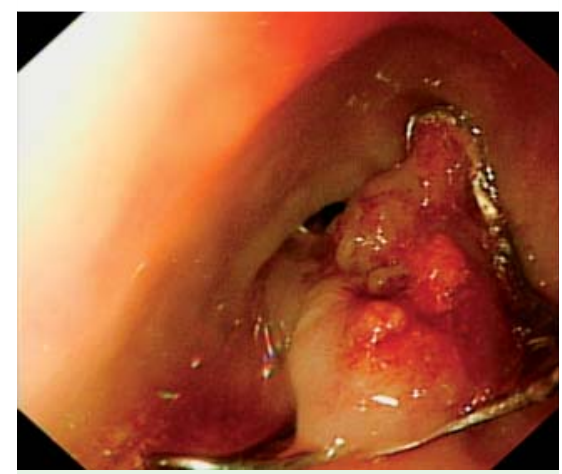

Fig. 5 Over-the-scope clip deployed, closing the perforation.

care, the patient was started on total parenteral nutrition. An elective endoscopy was performed under conscious sedation using fentanyl and midazolam. Endoscopy confirmed a $1-\mathrm{cm}$ tear at $40 \mathrm{~cm}$ from the incisors, confirming the site of esophageal perforation ( $\bullet$ Fig.2 and $\odot$ Fig. 3). An over-the-scope clip (Ovesco Endoscopy AG, Tübingen, Germany) was deployed after retracting the edges of the tear with a twin grasper forceps ( $\bullet$ Fig. 4 ). The perforation was closed successfully ( $\bullet$ Fig. 5). A nasojejunal feeding tube was placed endoscopically for enteral nutrition and total parenteral nutrition was discontinued. A repeat CT scan with oral contrast confirmed no further leak and an oral diet was started ( $\bullet$ Fig.6). Multiple loculated pleural effusions were managed with chest drains. The patient was discharged on antibiotic and proton pump inhibitor

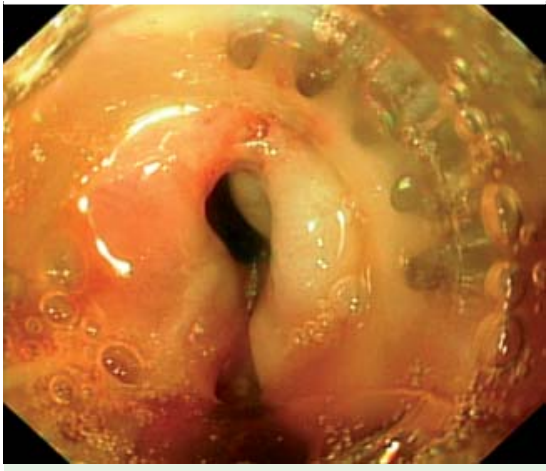

Fig.3 Esophageal perforation seen through the loaded over-the-scope clip.

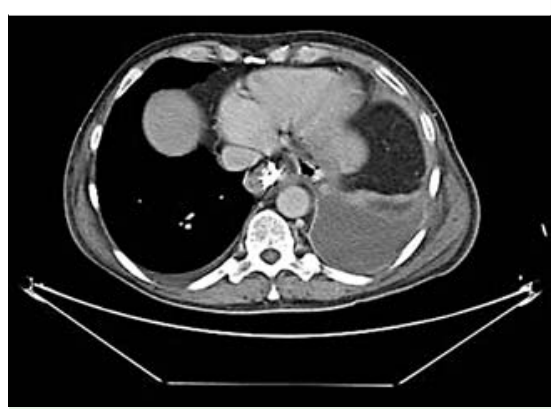

Fig. 6 Computed tomography scan following clip placement, confirming no esophageal leak.

therapy. He was asymptomatic on clinical follow-up at 3 months.

Acute esophageal perforation secondary to Boerhaave's syndrome with mediastinal complications has traditionally been managed surgically, with its associated morbidity $[1,2]$. Placement of covered metal or plastic stents is also widely practiced; however, this is associated with a significant need for re-intervention due to stent migration and persistent leaks [3-5]. Endoscopic closure of Boerhaave's syndrome with over-the-scope clips under conscious sedation is associated with significantly less morbidity, and facilitates early introduction of enteral nutrition and rapid recovery.

Endoscopy_UCTN_Code_TTT_1AO_2AI

\section{Competing interests: None}




\section{Sudheer Vinnamala', Vinod Menon², Ramesh Arasaradnam', Jayne A. Eaden', John L. H. Wong ${ }^{1}$, Jayan Mannath ${ }^{1}$}

${ }^{1}$ Department of Gastroenterology, University Hospitals Coventry and Warwickshire NHS Trust, Coventry, United Kingdom

${ }^{2}$ Department of Surgery, University Hospitals Coventry and Warwickshire NHS Trust, Coventry, United Kingdom

\section{References}

1 Prichard RS, Butt J, Al-Sarraf $N$ et al. Boerhaave's syndrome: a single centres management experience with fourteen cases of spontaneous oesophageal perforation. Endoscopy 2006; 38: Poster 56. doi: 10.1055/ s-2006-956858

2 Biancari F, D'Andrea V, Paone R et al. Current treatment and outcome of esophageal perforations in adults: systematic review and meta-analysis of 75 studies. World J Surg 2013; 37: 1051

3 Schubert D, Scheidbach $H$, Kuhn R et al. Endoscopic treatment of thoracic esophageal anastomotic leaks by using silicone-covered, self-expanding polyester stents. Gastrointest Endosc 2005; 61: 891 -896

4 Chung MG, Kang DH, Park DK et al. Successful treatment of Boerhaave's syndrome with endoscopic insertion of a self-expandable metallic stent: report of three cases and a review of the literature. Endoscopy 2001; 33: $894-897$

5 Eisendrath P, Devière J. Plastic stents in the treatment of benign esophageal conditions. Gastrointest Endosc 2008; 68: 402
Bibliography

Dol http://dx.doi.org/

10.1055/s-0034-1377535

Endoscopy 2014; 46: E481-E482

(c) Georg Thieme Verlag KG

Stuttgart · New York

ISSN 0013-726X

Corresponding author

Jayan Mannath, MD

Department of Gastroenterology

University Hospitals Coventry and Warwickshire

NHS Trust

Clifford Bridge Road

Coventry CV2 2DX

United Kingdom

jayan.mannath@uhcw.nhs.uk 